\title{
El docente universitario ante la emergencia educativa. Adaptación a las TIC en los procesos de enseñanza
}

\section{University teachers in the face of the educational emergency. Adaptation to ICT in teaching processes}

\author{
William Rodrigo Avendaño-Castro (c) \\ Universidad Francisco de Paula Santander, Cúcuta, Colombia \\ César Hernández-Suárez \\ Universidad Francisco de Paula Santander, Cúcuta, Colombia \\ Raúl Prada-Núñez \\ Universidad Francisco de Paula Santander, Cúcuta, Colombia
}

Open Access:

\section{Resumen}

Objetivo: Determinar en los docentes de educación superior, la adaptación a las TIC en la enseñanza durante la emergencia educativa provocado por el Covid 19. Método: Estudio cuantitativo enmarcado en el paradigma empírico analítico de carácter descriptivo transversal y diseño de campo no experimental. La muestra estuvo conformada por 96 docentes universitarios. Se utilizó un cuestionario autoadministrado de forma online con 24 ítems: dominio de las TIC, medios de comunicación adoptados y oportunidades de adaptación. Resultados: Los docentes tuvieron una adaptación luego de la experiencia vivida en la emergencia educativa por Covid-19. Por tanto, tienen la oportunidad de adaptación al entorno digital, flexibilidad a las TIC en la enseñanza y adaptación a la enseñanza con TIC. Conclusión: La adaptación es relativamente permanente y no desaparece una vez que finaliza el aprender, desaprender y volver aprender con TIC.

Palabras clave: Docente, universidad, Covid-19, adaptación, TIC.

\begin{abstract}
Objective: To determine the adaptation to ICT in teaching for higher education teachers during the educational emergency caused by Covid-19. Method: Quantitative study has been used, framed in the empirical analytical paradigm of a cross-sectional descriptive nature and non-experimental field design. The sample was made up of 96 teachers. A self-administered questionnaire was carried out online with 24 items: mastery in ICT, adopted media and adaptation opportunities. Results: The teachers went through an adaptation period after the experience of the educational emergency due to Covid-19. Therefore, they have the opportunity to adapt to the digital environment, flexibility to ICT in teaching and adaptation time to teaching with ICT. Conclusion: Adaptation is relatively permanent and does not disappear once learning, unlearning and relearning with ICT ends.
\end{abstract}

Keywords: Teacher, university, emergency, Covid-19, adaptation, ICT.

Recibido: $18-12-2020$

Cómo citar este artículo (APA): Avendaño-Castro, W., Hernández-Suárez, C. y Prada-Núñez, R. (2021). El docente universitario ante la emergencia educativa. Adaptación a las TIC en los procesos de enseñanza. Educación y Humanismo, 23(41), 27-46. https://doi.org/10.17081/eduhum.23.41.4354 



\section{Introducción}

Debido a la crisis sanitaria provocada por el Covid-19 y al aislamiento social obligatorio decretado por el Ministerio de Salud y Protección Social de Colombia (Resolución 385, 12 marzo 2020), el Estado colombiano ha venido adoptando diferentes medidas para hacer frente a la pandemia. Dentro de estas, se encuentra la Circular 021 (17 de marzo de 2020) del Ministerio de Educación Nacional en donde se brindan orientaciones para el desarrollo de procesos de planeación pedagógica y trabajo académico en casa. El resultado de ello es un cambio del modelo educativo presencial imperante, por otro que se configura en espacios de enseñanza remota y en línea, donde es indudable, el apoyo de las tecnologías de la información y las comunicaciones (TIC) dentro del proceso de enseñanza adoptado por los docentes. Sin duda, se ha requerido la adaptabilidad de los docentes a esta emergencia sanitaria.

La abundancia de herramientas, aplicaciones y entornos virtuales de aprendizaje dio a los docentes la libertad de elección, pero los obligó, junto con los estudiantes, a apropiarse rápidamente al aprendizaje desde casa. Aunque Guzmán, García, Espuny y Chaparro (2011) advierten que "la mayoría de los profesores son capaces de utilizar diferentes formatos de archivos, e integrar texto e imágenes en documentos. [...] Sin embargo, hay profesores con deficiencias en el uso de algunas de las herramientas TIC $[\ldots]^{\prime \prime}$ (p.11).

Cambiar a la educación remota y en línea puede ser una modalidad para reducir la propagación del Covid-19, pero esta tendencia, asimismo, generó retos, desafíos y oportunidades para los docentes, incluidos aquellos que carecen de habilidades TIC para enseñar en la educación basada en Internet.

Ante este nuevo escenario, no sólo se enfrentan al desafío de incorporar las tecnologías a los contenidos que sobreabundan en la web y formas de educación, sino que "la enseñanza universitaria, tradicionalmente, se ha fundamentado en un modelo metodológico centrado en el docente, con énfasis en la transmisión de contenidos y su reproducción por los alumnos, la lección magistral y el trabajo individual" (Cabero, López y Martínez, 2013, p.46).

Enseñar a través del uso de las TIC, demanda una serie de cambios que generan una ruptura con el modelo tradicional, al mismo tiempo es necesario identificar y reconocer cómo se puede llevar a cabo este proceso.

Algunos de los desafíos relacionados con el aprendizaje remoto (incluido el acceso a internet y manejo de dispositivos y programas) han llevado a ciertas escuelas y universidades a truncar sus clases. Sin embargo, en la creencia de una mayor dependencia del acceso a internet, algunos proveedores de servicios han alterado sus políticas, aumentado las velocidades de conexión a internet y eliminado los límites de datos para 
reducir la brecha digital en Colombia (Guzmán, 2017, p.45). También se han dispuestos más entonos virtuales para el aprendizaje.

La educación en su conjunto puede adaptarse a condiciones sorprendentemente diversas. Sin embargo, los actores educativos presentan diferentes formas de adaptabilidad. Esto significa que hay un rango de adaptación apropiado para cada representante del ámbito educativo en el contexto universitario. Esta adaptación al uso de las TIC podría permitir mejorar habilidades y capacidades de acuerdo con el contexto de cada actor en lugar de la suma del proceso de enseñanza-aprendizaje, porque las particularidades "requieren una reelaboración y adaptación cognitiva por parte del usuario" (Cabero, 2014, p.11).

Es imperioso recordar que existe una necesidad urgente de considerar la formación sobre las TIC del profesorado universitario para su adaptación a la enseñanza (Álvarez et al, 2011) puesto que, el papel real de la tecnología produce oportunidades muy diferentes que pueden estimular a "los maestros en un ambiente de aprendizaje con tecnología" (Farrell, 2002, p.44).

El docente debe poseer competencias digitales o en TIC, por lo que es fundamental la capacitación docente relacionadas con la incorporación de tecnologías en el aula según las expectativas, usos y necesidades de los actores (Hernández, Arévalo y Gamboa, 2016; Martín, Hernández y Mendoza, 2017, Gamboa, Hernández y Prada, 2018; Arévalo, García y Hernández, 2019). Se deberá tener en cuenta que los docentes coexisten con residentes digitales (White y Le Curne, 2011) e inmigrantes digitales (Prensky, 2001), grupos que con distinta formación, se adaptan a nuevos entornos por diferentes razones o motivos personales y profesionales.

Además, de acuerdo con Del Moral y Villalustre (2005), un factor de calidad en la docencia en los entornos virtuales puede "llevar a cabo una adaptación pedagógica en función de las características individuales de los estudiantes: funcionalidad; flexibilidad; agilidad; interactividad; usabilidad; accesibilidad; adaptabilidad y legibilidad" (p.18). De allí que las acciones debido a la mitigación y a la adaptación, permiten que, en promedio, las capacidades, pero crean interdependencia. Por lo tanto, un cambio en una parte de una función pedagógica apoyada en la tecnología afecta indirectamente las habilidades TIC del docente para afrontar en el futuro una educación remota de calidad. Este estudio brinda un adelanto en torno al uso de las TIC en tiempos de pandemia del Covid-19 y su adaptación entre los docentes en el contexto universitario.

Los desafíos y oportunidad de docentes para adecuarse a la enseñanza remota con uso de las TIC en tiempo de emergencia educativa y crisis, añaden un diferencial que condiciona la adaptación del ser humano y entiende que esta enseñanza en la educación superior no es solo una alternativa, sino que como "todo elemento que se considere de importancia para alcanzar logros, [...] se contextualiza y se adapta a los intereses particularidades de grupos humanos, se logra establecer una sociedad de la información acorde a los retos del mundo 
actual" (Jojao, 2014, p. 69), que incluye contextos virtuales y las zonas de posibilidades en los procesos de aprendizaje (Gómez, Hernández y Prada, 2020), de formación, de información y de conocimiento capaces de adaptarse a colectividades e individualidades.

Desde la perspectiva práctica, es necesario estudiar y reflexionar cobre la capacidad de adaptación de los docentes en contexto universitario, a las nuevas dinámicas de enseñanza con el uso del TIC en tiempos de crisis. Los hallazgos de estos análisis representan oportunidades en la comprensión de estos fenómenos para identificar necesidades existentes del medio e innovar en la educación desde las prácticas mediadas por TIC.

Por lo tanto, el objetivo de esta investigación es determinar en los docentes, la adaptación a las TIC en la enseñanza en tiempo de emergencia educativa por Covid-19, a través de la reestructuración de sus prácticas pedagógicas, estrategias académicas y modos de funcionamiento con el fin de dar continuidad a los cursos lectivos en los distintos programas académicos en la educación superior en el año 2020.

\section{La adaptación}

Los seres humanos tienen como atributo la flexibilidad y la adaptación al entorno vital, esto depende de una serie de factores independientes. En el caso de los organismos vivos, estos factores pudieran ser la presión, la temperatura, los alimentos, etcétera. En palabras de Jaume (2011) se confunde las adaptaciones con la adaptabilidad. Así, que algo sea una adaptación implica que es resultado de la selección natural, mientras que un rasgo puede ser adaptativo sin ser necesariamente una adaptación.

Aunque para Loredo (2004) al hacer referencia de la teoría de la selección orgánica de Baldwin (1861-1934) señala que "la selección natural es un caso límite de la selección orgánica: aquel en el cual el comportamiento no puede desempeñar ningún papel adaptativo debido a la aparición de cambios radicales en el medio tales como catástrofes ecológicas" (p.189). Además, argumenta que, en los demás casos, la selección es una consecuencia de la adaptación, y no al revés por lo que Baldwin hablaba a veces de selección indirecta.

Otro autor que basó sus ideas en el trabajo Baldwin fue Jean Piaget (1969), con la finalidad de explicar el desarrollo de la inteligencia humana y la deducción basada en las raíces biológicas, para quien los mecanismos de desarrollo son mediante el movimiento a través de etapas sucesivas que dependen de la retroalimentación del entorno estimulante y consideró que la similitud (el efecto del medio ambiente en el organismo), conduce a la adaptación del organismo (cambio flexible), por lo que la socialización, como estrategia adaptativa humana, permite la adaptación.

Así pues, en opinión de Piaget (1969), la inteligencia es una función básica que ayuda a un individuo a adaptarse al entorno, que incluye la organización interna de la información recibida y la adaptación al entorno de uno. Por ello, es inherente que se integre el conocimiento adquirido en un sistema de conocimiento existente. Esta actividad es 
adaptarse al entorno, que tiene lugar de dos maneras: A través de la asimilación y/o la adaptación.

Por ello, Piaget (1969) sostiene que los organismos mantienen interacciones con el medio, que tienden a adaptarse, para mantener un estado de equilibrio con el mismo. La inteligencia o el conocimiento, es una forma de adaptación de un organismo complejo a un medio complejo, a sus experiencias que "... comporta aptitud para adaptarse, para adaptar, incluso para adoptar" (acoger otro medio cambiando a él) (Morin, 1980, p. 48). Por tanto, el ser humano (docente) funciona como un todo para adaptarse ante cualquier circunstancia del medio, incluido, la adaptación al uso de las tecnologías.

\section{El docente ante la emergencia educativa y la enseñanza remota}

Antes de la pandemia, algunas escuelas y universidades de modalidad presencial contaban con docentes formados para enseñar a los estudiantes de forma virtual o remota, pero en su gran mayoría, los docentes no tenían ningún tipo de experiencia en estos tipos de formación.

Durante la pandemia, los docentes y estudiantes permanecen fuera de las aulas, y las instituciones educativas intentan proporcionar algún tipo de enseñanza con mediación tecnológica que le permita avanzar en los procesos educativos. En el contexto universitario, dependerá de "la flexibilidad de la organización del trabajo que sustenta la búsqueda de la adaptabilidad constante en modelos de producción basados en información, conocimiento..." (Arancibia, 2011, p. 43), para enseñar de manera remota e "impulsados por la urgencia de implementar una solución factible y práctica a las crisis, con educadores que utilizan tecnología familiar" (Goh y Sandars, 2020).

Hay que considerar que este tipo de enseñanza proviene, primero, de la educación a distancia, que "es un método de educación que difiere de la educación presencial, mientras que el aprendizaje abierto describe la naturaleza de la educación ofrecida, sea ésta presencial o a distancia" (García, 2002, p. 14). La educación a distancia se refiere a una metodología de enseñanza diferenciada en el tiempo, en el espacio o en ambas a la vez. Garduño (2005, p. 4), afirma que "...el término educación a distancia involucra desde el campo de estudio de la educación por correspondencia (basada en texto impreso), hasta la educación virtual (o educación en línea), que se apoya en medios generados a través de TIC".

Segundo, se observa el uso de internet como medio principal de interacción de la educación a distancia. Al respecto, Lassos, Munévar, Rivera, y Sabogal (2017) señalan que "la educación virtual es la evolución de la educación a distancia, lograda por el desarrollo y fortalecimiento de la relación educación y tecnología" (p. 22). Sobre este particular, Garduño (2005) define la educación virtual, como un método no presencial, basado en las TIC "...utilizando como herramienta fundamental el soporte informático, sin prescindir ni atenuar 
la relación profesor-alumno, pues la comunicación se mantiene y fomenta mediante la red" (p.7).

Por consiguiente, el docente ante la emergencia educativa imparte una educación mediada por las TIC y debe superar algunos retos, tanto en sus competencias TIC como en sus habilidades para enseñar con tecnología. Goh \& Sandars (2020) dicen que en los tutores que se adaptan, para facilitar su aprendizaje individual autorregulado, existe la oportunidad de reducir el tiempo para el desarrollo de la competencia individual y disminuir el tiempo requerido para la interacción cara a cara. Esto podría servir a la educación en la virtualidad o educación virtualizada, debido a que la enseñanza desde "lo virtual... sugiere acción y creación, en este caso, pedagógica, que indica un ejercicio didáctico constante para la estructuración de estrategias metodológicas que permitan el proceso formativo" (Lassos, Munévar, Rivera y Sabogal, 2017, p. 23).

\section{El docente ante las TIC}

La educación es un sistema en el que todas las partes tienen su propia función directa o indirecta, pero siempre es importante entender primero, que existe un sincretismo entre el docente, el aprendizaje y las tecnologías como elementos del sistema educativo y segundo, que el docente es un agente adaptativo al medio de la organización donde opera. Por ello, para entender el entorno, es importante percibir todo el sistema de fondo que está fuera de la educación y en el que opera una persona.

En el primer punto, el docente entiende que, en la era digital, el aprendizaje concibe a "la mente humana como una red que se adapta al entorno. Por lo tanto, el aprendizaje sería el proceso de formación de redes a través de conexiones entre distintos nodos, y el conocimiento residiría en dichas redes" (Sánchez, Costa, Mañoso, Novillo y Pericacho, 2019, p. 115). Esto se fundamenta en la teoría conectivista de Siemens (2004). Segundo, el docente de educación superior, donde la universidad como organización es un sistema abierto, mantiene una constante adaptabilidad y capacidad de adaptarse y acoplarse a los cambios. Este "proceso de adaptación en el cual el ser humano debe modificar el comportamiento para acoplarse a nuevos procesos para desarrollar la capacidad para acomodarse de forma eficiente y realizar las funciones sin que afecte las actividades" (Guzmán, 2018, p. 7).

Entonces, el uso de las TIC en la organización posibilita al docente ampliar sus oportunidades, tales como organizar sus cursos en línea, facilitar las interacciones de los actores en medio virtual, así como aprender-aprender en línea, realizar una adaptación y respuesta a las demandas educativas y de las relaciones con sus estudiantes para conseguir los fines del proceso formativo. En consecuencia, él docente ante las TIC no solo es quien proporciona la enseñanza mediada, sino que también es un aprendiz que tiene la posibilidad junto a sus estudiantes de "autorregular su aprendizaje, [...] reestructuran sus contextos para alcanzar las metas, controlan el uso del tiempo, utilizan métodos propios de 
autoevaluación, atribuyen sus resultados a sus propios procesos y capacidades y adaptan sus métodos y estrategias" (Díaz, Pérez, Valenzuela, Muñoz, Rivas y Salas, 2010, p. 790).

De este modo, el docente "se ha visto desafiado a ubicar resoluciones creativas e innovadoras, actuando y aprendiendo sobre la marcha, demostrando capacidad de adaptabilidad y flexibilización de los contenidos y diseños de los cursos para el aprendizaje en las distintas áreas de formación" (UNESCO-IESALC, 2020, p. 21). Según Barberousse (2008) "los procesos de adaptación y cambio siempre se han dado hacia el equilibrio y motivados no por el mismo sistema, sino sólo como respuesta a lo que lo amenaza (como lo plantea la propuesta piagetiana)" (p.102).

\section{Metodología}

\section{Tipo de investigación}

Corresponde a un estudio cuantitativo no experimental y transversal enmarcado en el paradigma empírico-analítico. La investigación fue de carácter descriptivo y diseño de campo con métodos cuantitativos. Por ello, se investigó en los perfiles de docentes de educación superior para determinar la oportunidad de adaptación al uso de las TIC durante la enseñanza en tiempos de la pandemia y la emergencia educativa provocada por el Covid19.

\section{Población y Muestra}

La población estuvo constituida por 126 docentes pertenecientes a una Facultad de Ciencias de Salud en una universidad colombiana de naturaleza pública. Los datos poblacionales se lograron a través de una encuesta online para docentes que han venido utilizando la enseñanza mediada por TIC virtual en tiempo de COVID-19. Para obtener el tamaño de la muestra en poblaciones finitas, se empleó la siguiente formula estadística:

$n=\frac{Z c^{2} \times P \times Q}{E^{2} \times(N-1)+Z c^{2} \times P \times Q}$

Donde: $\mathrm{Zc}=95 \%$ o 1.96 es el nivel de certeza, bajo la curva normal; $\mathrm{P}=0,5$ es la probabilidad de éxito; $Q=0,5$ es la probabilidad de fracaso; $E=5 \%-0,05$ es el nivel de error; y $\mathrm{N}=126$ es la población (Bernal, 2010). El resultado obtenido fue de 96 sujetos. 


\section{Instrumento para recolección de datos}

Para la recopilación de la información de la investigación, se elaboró un cuestionario, el cual fue validado a través de juicio de expertos. El instrumento fue autoadministrado de manera online estructurado con 24 preguntas con varias opciones de respuesta. Se creyó favorable organizarlo en tres bloques que mostraran todos los aspectos afines con los objetivos planteados.

El primer bloque lo conforman las preguntas de carácter general (edad, sexo, grado de estudios, años de experiencia docente en educación superior, entre otras), por lo que se identificó como "datos personales e institucionales". El segundo bloque estuvo referido a la formación en TIC (competencias TIC) y los medios y herramientas utilizados durante la pandemia para finalizar los cursos impartidos por los encuestados. El último bloque se enfocó en examinar la adopción y adaptación al uso de las TIC y su mediación en la enseñanza. Para estimar la validez de contenido del cuestionario se empleó el juicio de expertos.

\section{Procedimiento y análisis de datos}

Los cuestionarios se aplicaron de manera online, durante el mes de abril y mayo de 2020. El criterio de selección fue que el docente impartiera clases en modalidad presencial por la estructura teórico-práctico que tienen involucradas las asignaturas. De allí que la selección de dicha facultad fue porque sus programas formativos se realizan bajo la presencialidad y la muestra era representativa para el presente estudio. Respondieron a los cuestionarios un total de 315 profesores, de los cuales 126 pertenecen a la facultad de ciencias de la salud (población objeto de estudio), lo que representó un $40 \%$ de encuestas válidas y la cantidad necesaria para cubrir el tamaño de la muestra obtenida de 96 sujetos. Los datos recolectados fueron organizados y tabulados en hojas de cálculo del programa Excel. Se efectúo una exploración descriptiva en el cuestionario en el que se incluyeron estadísticos como la frecuencia para el análisis e interpretación de los datos.

\section{Resultados}

A continuación, se hace una descripción de los resultados obtenidos con la aplicación del cuestionario sobre oportunidades de adaptación a las TIC en docentes de educación superior ante la emergencia educativa generada por el coronavirus Covid-19.

En este estudio se determinó que el $61.5 \%$ de los docentes encuestados era de sexo femenino y un $38.5 \%$ masculino. En cuanto a la edad, se evidenció que el $36.5 \%$ se encuentra en un rango de 41 a 50 años. Otro $31.3 \%$ está entre las edades de 31 a 40 años, mientras que el $25 \%$ es mayor de 50 años. El resto de los docentes se localizan entre las 
edades de 26 a 30 años (5.2\%) y menores de 25 años (2.1\%).

En cuanto al nivel educativo, el $43.8 \%$ posee un grado de estudios completado de especialista; el $41.7 \%$ tiene título de magíster y el $10.4 \%$ posee doctorado. El $4.2 \%$ es profesional universitario.

En lo referente a los años de experiencia docente en educación superior, se conoció que el $40.6 \%$ tiene un tiempo en la educación superior de entre cero (0) a 5 años; un 25\% manifestó tener entre 6 a 10 años de experiencia de docencia universitaria. El resto de los encuestados, manifiestan que los años de práctica que han ejercido la enseñanza superior está entre 11 a 15 años (12.5\%); entre 16 a 20 años (9.4\%) y más de 20 años el 12.5\%.

En lo referente al uso de plataformas tecnológicas para la gestión del aprendizaje de los estudiantes, se determinó que el $96.6 \%$ utiliza la plataforma de la universidad; el $1 \%$ manifestó que no usa la plataforma de la universidad y otro $2.1 \%$ dijo que usa el servicio de videoconferencia desarrollado por Google denominado Meet, para la gestión del aprendizaje de los estudiantes.

Una vez estimada esta información sobre datos poblacionales e institucionales se tomaron en cuenta aspectos relacionados con al acceso a la tecnología, internet herramientas de comunicación y colaboración.

Se observó que el $100.0 \%$ de los encuestados cuenta en su casa con acceso a computadora, laptop u otro dispositivo con internet para continuar el ejercicio profesional durante la pandemiaCovid-19. El $99.0 \%$ tiene acceso a herramientas de comunicación asincrónica y sincrónica y un $96.9 \%$ a herramientas de colaboración asincrónica y sincrónica.

También se indagó sobre la percepción de los docentes en el dominio de las TIC para afrontar el desafío de la enseñanza remota (ver tabla 1).

\section{Tabla 1.}

Percepción de los docentes en el de dominio de las TIC durante la emergencia educativa

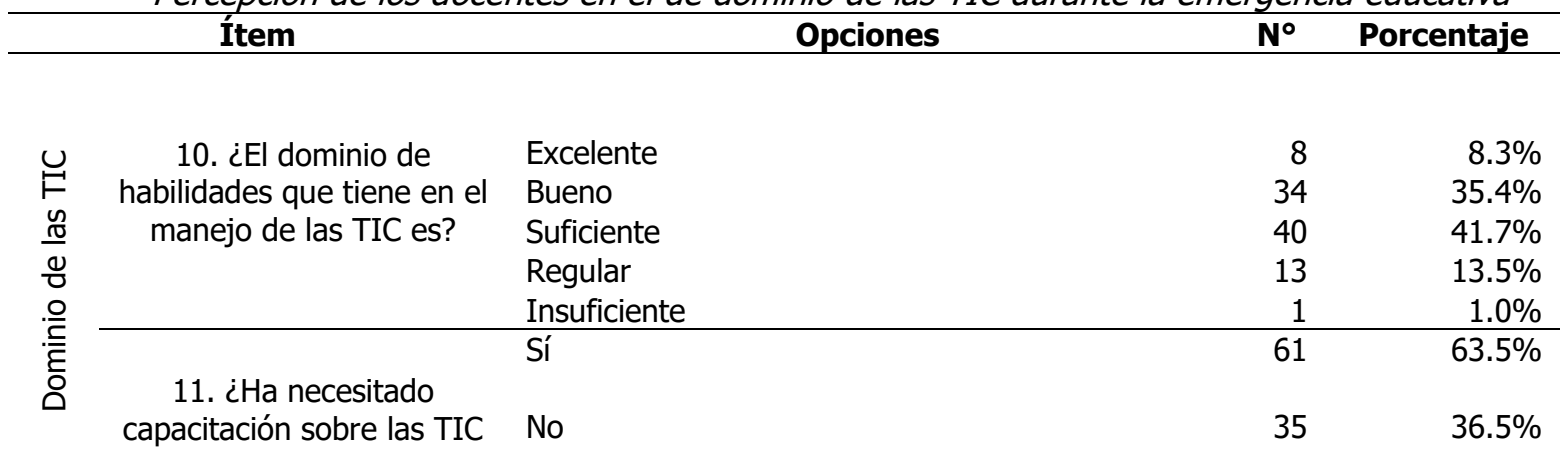

Fuente: Elaboración propia a partir del instrumento de Encuesta realizado para la investigación (2021) 
Los datos registrados la tabla 1, muestran que el ítem 10, reveló que el $41.0 \%$ de los docentes encuestados dijeron que disponen un dominio suficiente de las habilidades en el manejo de las TIC. Mientras que un 35.4\% precisaron que el dominio de las TIC es bueno y un $8.3 \%$ dijo que excelente. No obstante, existe un $13.5 \%$ que opina que sus dominios son regulares y un $1.0 \%$ dijo que es insuficiente. En lo que se refiere al ítem 11, cabe destacar que el $63.5 \%$ ha necesitado capacitación sobre las TIC y el $36.5 \%$ no lo ha necesitado.

Los resultados conforme al ítem 10, muestran los mayores porcentajes en las alternativas excelente, bueno y suficiente, para un acumulado del $85.4 \%$ de los consultados respecto del dominio de habilidades en el manejo de las TIC. Eso evidencia que los docentes se sienten capaces y competentes frente al manejo de las TIC, lo que implica su entendimiento, usos y aplicación. Este es un indicador positivo de la adaptación de la enseñanza a las TIC, y que pueden tener efectos positivos sobre lo pedagógico, lo curricular, lo didáctico y lo evaluativo.

En la tabla 2 se muestra la frecuencia de los medios adoptados por los profesores durante la emergencia educativa para comunicarse con los estudiantes.

\section{Tabla 2.}

Medios de comunicación adoptados ante la emergencia educativa

\begin{tabular}{|c|c|c|c|c|}
\hline & Ítem & Opciones & $\mathbf{N}^{\circ}$ & Porcentaje \\
\hline \multirow{14}{*}{ 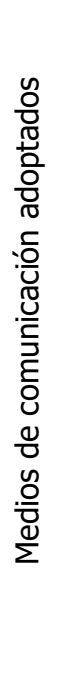 } & \multirow{9}{*}{$\begin{array}{c}12 \text { ¿Medios utilizados } \\
\text { para comunicarse con } \\
\text { los estudiantes para } \\
\text { enfrentar la } \\
\text { emergencia educativa } \\
\text { causada por el Covid- } \\
19 ?\end{array}$} & Blogs & 8 & $8.3 \%$ \\
\hline & & Correo electrónico & 94 & $97.9 \%$ \\
\hline & & Chat & 68 & $70.8 \%$ \\
\hline & & Página personal & 10 & $10.4 \%$ \\
\hline & & Plataformas educativas & 80 & $83.3 \%$ \\
\hline & & Facebook & 8 & $8.3 \%$ \\
\hline & & Twitter & 2 & $2.1 \%$ \\
\hline & & Mensajería instantánea (WhatsApp, Telegram) & 74 & $77.1 \%$ \\
\hline & & Servicio de videotelefonía (Skype, Meet) & 16 & $16.7 \%$ \\
\hline & \multirow{5}{*}{$\begin{array}{l}\text { 13. Durante la actual } \\
\text { crisis de la pandemia } \\
\text { Covid-19, ¿Usted } \\
\text { adoptó } \\
\text { pedagógicamente las } \\
\text { TIC en sus cursos } \\
\text { para? (Opciones } \\
\text { múltiples) }\end{array}$} & Comunicación con estudiantes y colegas & 91 & $94.8 \%$ \\
\hline & & $\begin{array}{l}\text { Evaluación y retroalimentación de aprendizaje de } \\
\text { los estudiantes. }\end{array}$ & 89 & $92.7 \%$ \\
\hline & & $\begin{array}{l}\text { Creación y producción de materiales multimedia, } \\
\text { web o de presentación. }\end{array}$ & 63 & $65.6 \%$ \\
\hline & & $\begin{array}{l}\text { Aplicación de tecnologías interactivas digitales } \\
\text { para mejorar el aprendizaje. }\end{array}$ & 57 & $59.4 \%$ \\
\hline & & No aplica. & 1 & $1.0 \%$ \\
\hline
\end{tabular}

De los datos obtenidos en el ítem 12, se puede apreciar que fue el correo electrónico, 
con un $97.9 \%$, el medio más usado por los docentes ante la emergencia educativa para comunicarse con los estudiantes, seguido por las plataformas educativas (LMS, Moodle) con un $83.3 \%$, el blog $(8.3 \%)$ y página personal del docente $(10.4 \%)$ para poder dar a conocer las instrucciones a las asignaciones y actividades a realizar.

También se observa comunicación sincrónica a través de la mensajería instantánea (77.1\%) con las aplicaciones de WhatsApp y Telegram. Además, se usó el chat de la plataforma educativa (70.8\%) y, el servicio de videotelefonía (Skype y Meet) en un 16.7\%. Es importante destacar que se e evidenció poco uso de las redes sociales: un $8.3 \%$ en Facebook y un $2.1 \%$ por Twitter.

En lo referente al ítem 13, se conoció que los docentes adoptaron pedagógicamente las TIC para comunicación con estudiantes y colegas (94.8\%) lo cual concuerda con los hallazgos del ítem 11.

De igual manera, se demostró que adoptaron las TIC para aplicar evaluación y retroalimentación de aprendizaje con los estudiantes (92.7\%), así como para la creación y producción de materiales multimedia, web o de presentación (65.6\%) y aplicación de tecnologías interactivas digitales para mejorar el aprendizaje (59.4\%). Aunque uno de ellos dijo que no aplica (1.0\%). Este último dato concuerda con el resultado del ítem 10, donde uno de los encuestados dijo que su dominio de las TIC era insuficiente.

Bajo esta perspectiva, estos resultados posibilitan actuaciones hacia la flexibilidad en el trabajo requeridos al campo específico del conocimiento con el uso de las TIC, y, por tanto, de contar con las nociones necesarias para integrar las tecnologías de información y comunicación para la enseñanza remota en los tiempos de emergencia educativa y luego de dicha emergencia.

A continuación, se muestra la tabla 3, que describe la oportunidad de adaptación a las TIC por parte de los profesores.

Tabla 3.

Oportunidad de adaptación a las TIC

\begin{tabular}{|c|c|c|c|c|c|c|c|}
\hline \multicolumn{3}{|c|}{ Ítem } & \multicolumn{5}{|c|}{ Opciones } \\
\hline & & & $\begin{array}{c}\text { Seguramente } \\
\text { probable }\end{array}$ & $\begin{array}{l}\text { Bastante } \\
\text { probable }\end{array}$ & $\begin{array}{c}\text { Algo } \\
\text { probable }\end{array}$ & $\begin{array}{c}\text { Poco } \\
\text { probable }\end{array}$ & $\begin{array}{c}\text { Casi } \\
\text { Improbable }\end{array}$ \\
\hline 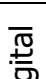 & $\begin{array}{l}\text { 14. ¿Considera probable adaptarse } \\
\text { a otros recursos TIC, para }\end{array}$ & $F$ & 63 & 27 & 6 & 0 & 0 \\
\hline 吾 & $\begin{array}{l}\text { conseguir acceso a otras } \\
\text { herramientas que ayuden a } \\
\text { continuar el ejercicio profesional } \\
\text { postpandemia Covid-19? }\end{array}$ & $\%$ & $65.6 \%$ & $28.1 \%$ & $6.3 \%$ & $0.0 \%$ & $0.0 \%$ \\
\hline $\begin{array}{l}\overline{0} \\
\text { ㄷํㅇ }\end{array}$ & $\begin{array}{l}\text { 15. Desde su punto de vista, } \\
\text { ¿adaptarse al uso de las TIC le }\end{array}$ & $F$ & 71 & 20 & 4 & 1 & 0 \\
\hline $\begin{array}{l}\text { 華 } \\
\frac{0}{0} \\
\frac{\pi}{0} \\
\end{array}$ & $\begin{array}{l}\text { ayudará a fortalecer su quehacer } \\
\text { pedagógico luego de la pandemia } \\
\text { Covid-19? }\end{array}$ & $\%$ & $74.0 \%$ & $20.8 \%$ & $4.2 \%$ & $1.0 \%$ & $0.0 \%$ \\
\hline
\end{tabular}




\begin{tabular}{|c|c|c|c|c|c|c|c|}
\hline & \multirow{2}{*}{$\begin{array}{l}\text { 16. ¿Considera probable asimilar } \\
\text { el acceso y uso de repositorios } \\
\text { digitales y bases de datos para } \\
\text { fortalecer su quehacer pedagógico } \\
\text { luego de la pandemia Covid-19? }\end{array}$} & $\mathrm{F}$ & 59 & 28 & 9 & 0 & 0 \\
\hline & & $\%$ & $61.5 \%$ & $29.2 \%$ & $9.4 \%$ & $0.0 \%$ & $0.0 \%$ \\
\hline & \multirow{2}{*}{$\begin{array}{l}\text { 17. ¿Considera probable adoptar } \\
\text { el uso de herramientas de } \\
\text { comunicación asincrónicas luego } \\
\text { de la experiencia para enfrentar la } \\
\text { emergencia educativa generada } \\
\text { por la pandemia del Covid-19? }\end{array}$} & $\mathrm{F}$ & 64 & 28 & 4 & 0 & 0 \\
\hline & & $\%$ & $66.7 \%$ & $29.2 \%$ & $4.2 \%$ & $0.0 \%$ & $0.0 \%$ \\
\hline & \multirow{2}{*}{$\begin{array}{l}\text { 18. ¿Considera probable adoptar } \\
\text { el uso de herramientas de } \\
\text { comunicación sincrónicas luego de } \\
\text { enfrentar la emergencia } \\
\text { educativa? }\end{array}$} & $\mathrm{F}$ & 66 & 27 & 3 & 0 & 0 \\
\hline & & $\%$ & $68.8 \%$ & $28.1 \%$ & $3.1 \%$ & $0.0 \%$ & $0.0 \%$ \\
\hline \multirow{8}{*}{ 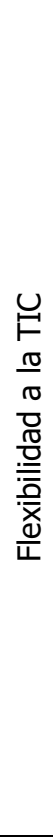 } & \multirow{2}{*}{$\begin{array}{l}\text { 19. ¿En qué medida de } \\
\text { probabilidad el uso de la TIC es } \\
\text { una oportunidad que considera } \\
\text { utilizar luego de la emergencia } \\
\text { educativa? }\end{array}$} & $\mathrm{F}$ & 67 & 27 & 2 & 0 & 0 \\
\hline & & $\%$ & $69.8 \%$ & $28.1 \%$ & $2.1 \%$ & $0.0 \%$ & $0.0 \%$ \\
\hline & \multirow{2}{*}{$\begin{array}{l}\text { 20. ¿Considera probable que la } \\
\text { institución en la que labora tenga } \\
\text { una visión de cómo los } \\
\text { estudiantes y los profesores } \\
\text { deberían utilizar pedagógicamente } \\
\text { las TIC luego de la emergencia } \\
\text { educativa? }\end{array}$} & $\mathrm{F}$ & 41 & 29 & 22 & 4 & 0 \\
\hline & & $\%$ & $42.7 \%$ & $30.2 \%$ & $22.9 \%$ & $4.2 \%$ & $0.0 \%$ \\
\hline & \multirow{2}{*}{$\begin{array}{l}\text { 21. En su opinión ċla institución en } \\
\text { donde labora promoverá espacios } \\
\text { para discutir y planificar sobre el } \\
\text { uso de TIC luego de la crisis del } \\
\text { Covid-19? }\end{array}$} & $F$ & 32 & 33 & 25 & 6 & 0 \\
\hline & & $\%$ & $33.3 \%$ & $34.4 \%$ & $26.0 \%$ & $6.3 \%$ & $0.0 \%$ \\
\hline & \multirow{2}{*}{$\begin{array}{l}\text { 22. Desde su perspectiva ¿Será } \\
\text { probable una adaptación al uso de } \\
\text { las TIC como apoyo didáctico en } \\
\text { los procesos de enseñanza luego } \\
\text { de la emergencia educativa? }\end{array}$} & $\mathrm{F}$ & 68 & 26 & 2 & 0 & 0 \\
\hline & & $\%$ & $70.8 \%$ & $27.1 \%$ & $2.1 \%$ & $0.0 \%$ & $0.0 \%$ \\
\hline \multirow{9}{*}{ 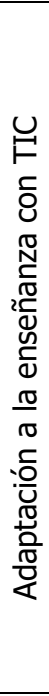 } & \multirow{2}{*}{$\begin{array}{l}\text { 23. ¿Piensa usted que es probable } \\
\text { la adaptación al uso de las TIC es } \\
\text { significativa para afrontar los } \\
\text { procesos de enseñanza y } \\
\text { aprendizaje luego del Covid-19? }\end{array}$} & $\mathrm{F}$ & 72 & 22 & 2 & 0 & 0 \\
\hline & & $\%$ & $75.0 \%$ & $22.9 \%$ & $2.1 \%$ & $0.0 \%$ & $0.0 \%$ \\
\hline & $\begin{array}{l}\text { 24. Luego de haber desarrollado } \\
\text { su curso durante la pandemia } \\
\text { Covid-19, ¿cuál será la } \\
\text { oportunidad probable para } \\
\text { adaptarse y adoptar los siguientes } \\
\text { aspectos? }\end{array}$ & & & & & & \\
\hline & \multirow{2}{*}{$\begin{array}{l}\text { 24.1) Organización del curso en } \\
\text { línea }\end{array}$} & $\mathrm{F}$ & 27 & 48 & 19 & 2 & 0 \\
\hline & & $\%$ & $28.1 \%$ & $50.0 \%$ & $19.8 \%$ & $2.1 \%$ & $0.0 \%$ \\
\hline & \multirow{2}{*}{$\begin{array}{l}\text { 24.2) Utilización de contenidos } \\
\text { multimedios }\end{array}$} & $F$ & 30 & 46 & 18 & 2 & 0 \\
\hline & & $\%$ & $31.3 \%$ & $47.9 \%$ & $18.8 \%$ & $2.1 \%$ & $0.0 \%$ \\
\hline & \multirow{2}{*}{$\begin{array}{l}24.3) \text { Curación de recursos y } \\
\text { materiales en línea para lograr el } \\
\text { aprendizaje de los estudiantes }\end{array}$} & $\mathrm{F}$ & 26 & 42 & 24 & 3 & 1 \\
\hline & & $\%$ & $27.1 \%$ & $43.8 \%$ & $25.0 \%$ & $3.1 \%$ & $1.0 \%$ \\
\hline
\end{tabular}


Los resultados que se muestran en la tabla 3 revelaron que en el ítem 14, el 93.7\% de los encuestados manifestó que, luego de la pandemia Covid-19, es bastante y seguramente probable considerar adaptarse a otros recursos TIC para conseguir acceso a otras herramientas que ayuden a su ejercicio profesional. El resto, dijo que era algo probable (6.3\%).

En el caso del ítem 15, el 94.8\% opina que, luego de la pandemia, adaptarse al uso de las TIC ayudará bastante y seguramente probable para fortalecer su quehacer pedagógico. El resto, señaló que ayudará algo probable (4.2\%) y poco probable (1.0\%).

En el ítem 16, se evidenció que el $90.6 \%$ de los profesores reflexionan que luego de la pandemia Covid-19, es bastante y seguramente probable que asimilen o se adapten al acceso y uso de repositorios digitales y bases de datos para fortalecer su quehacer pedagógico. El otro $9.4 \%$ dijo que es algo probable que lo considere.

Con relación al ítem 17, el 95.8\% declaró que luego de la experiencia para enfrentar la emergencia educativa generada por la pandemia del Covid-19, consideran bastante y seguramente probable adoptar el uso de herramientas de comunicación asincrónicas. El resto dijo que es algo probable que lo haga. Por otro lado, al examinar el ítem 18, se constató que el $\mathbf{9 6 . 9 \%}$ de los encuestados luego de enfrentar la emergencia educativa consideran bastante y seguramente probable adoptar el uso de herramientas de comunicación sincrónicas. El otro $3.1 \%$ mencionó que es algo probable que utilice estas herramientas.

En cuanto al ítem 19, se verificó que el 97.9\% de los educadores encuestados considera bastante y seguramente probable una oportunidad de usar las TIC luego de utilizarla en la emergencia educativa del Covid-19. Otro $2.1 \%$ expresó que algo probable.

Por otro lado, en el ítem 20, se confirmó que el $72.9 \%$ de los profesores piensa que es bastante y seguramente probable que la universidad donde labora tenga una visión de cómo los estudiantes y profesores deberían utilizar pedagógicamente las TIC luego de la experiencia vivida durante la pandemia del Covid-19. Otro $22.9 \%$ dijo que será algo probable que tenga esa visión de cómo utilizar las TIC pedagógicamente y 4.2\% manifestó que es poco probable que la universidad tenga dicha visión.

En esa misma dirección, se determinó a través del ítem 21 , que el $67.7 \%$ de los encuestados cree que, luego de la emergencia, es bastante probable o seguramente probable que la institución promoverá espacios para discutir y planificar sobre el uso de TIC, mientras que un $26.0 \%$ cree que será algo probable y el resto opina que será poco probable (6.3\%).

En lo relativo al ítem 22, se comprobó que el $97.9 \%$ de los profesores encuestados creen que de acuerdo con su perspectiva será bastante y seguramente probable que hagan una 
adaptación al uso de las TIC como apoyo didáctico en los procesos de enseñanza luego de la experiencia vivida durante la emergencia educativa. Otro $2.1 \%$ dijo que será algo probable.

Con respecto al ítem 23, probó que $97.9 \%$ de los encuestados piensa que luego del Covid-19 es bastante y seguramente probable que se adapten al uso de las TIC de manera significativa para afrontar los procesos de enseñanza y aprendizaje. Entre tanto, el $2.1 \%$ declaró que será algo probable.

A propósito del ítem 24, se conoció en los profesores que luego de haber desarrollado su curso de manera remota durante la pandemia Covid-19, creen que será bastante y seguramente una oportunidad de organizar sus cursos en línea (78.1\%). Así como una ocasión bastante y seguramente probable para utilizar contenidos multimedia $(79.2 \%)$ y hacer curación de recursos y materiales en línea para lograr el aprendizaje de los estudiantes (70.9\%).

\section{Discusión y conclusión}

El análisis de los resultados acerca de las oportunidades de los docentes en la adaptación a las TIC, evidencia que se concuerda con el planteamiento de Cabero (2014) quien dice que se demanda una reelaboración y adaptación cognitiva por parte del individuo. Se infiere que esta adaptación ha sido un proceso de adaptabilidad a la enseñanza en la era digital y a los cambios constantes del trabajo docente.

La adaptación en la enseñanza con TIC o en la enseñanza remota, así como en la educación virtual, es una respuesta adecuada a situaciones desafiantes para actuar y aprender sobre la marcha, demostrando adaptabilidad y flexibilidad en los contenidos y diseños de los cursos para aprender un conjunto habilidades en tecnologías como se demostró en la (tabla 1), que se deduce que los docentes han venido adaptándose a las nuevas tendencias educativas, entre ellas el uso de las TIC.

Esto concuerda con Del Moral y Villalustre (2005) quienes aducen que llevar a cabo una adaptación pedagógica en función de las características individuales de los estudiantes incluye flexibilidad, agilidad, interactividad, usabilidad, accesibilidad, adaptabilidad y legibilidad. Estas características posibilitan un dominio para adquirir, procesar, almacenar, producir, recuperar, presentar y difundir cualquier tipo de información integrando todos los medios disponibles que permitan procesar y difundir información con fines didácticos y prácticos promoviendo el acceso al conocimiento por medio del uso de las TIC como se afirmó en la (tabla 2).

El hecho de que los profesores adoptaran diversos medios durante la emergencia educativa, tales como: correo electrónico, plataformas educativas, blogs, página personal, redes sociales (Facebook, Twitter), mensajería instantánea (WhatsApp, Telegram), 
videotelefonía (Skype y Meet) para la comunicación con estudiantes y colegas, así como para evaluación y retroalimentación del aprendizaje, creación y producción de materiales multimedia, web o de presentación y aplicación de tecnologías interactivas digitales para mejorar el aprendizaje, coincide con lo expresado por Jojao (2014), quien sostiene que la adaptación se da por intereses particulares de grupos humanos y concuerda con el planteamiento de Gómez, Hernández y Prada (2020) quienes señalan que existe una zona de posibilidades en los procesos de aprendencia para satisfacer las demandas desafiantes del contexto virtual.

De todo lo anterior, se desprende que la enseñanza con el uso de las TIC en la era digital requiere adaptación y desarrollo de competencias para los educadores, por ello se presenta las siguientes oportunidades luego de la experiencia vivida en la emergencia educativa por el Coivd-19, a saber:

Adaptación al entorno digital. Corresponde al mecanismo que conlleva a un proceso de aprendizaje continuo dentro del entorno digital para hacer cambios específicos o estructurales. Es una acomodación en función de las TIC para estimular el aprendizaje en red, así como el desarrollo de aptitudes de alfabetización en TIC (trabajo móvil, procesamientos de textos, edición de imágenes y manejo de diversos dispositivos electrónicos, acceso a repositorios, entre otras).

En el caso del docente, la adaptación al entorno digital se materializa en las capacidades para una efectiva enseñanza en línea: LMS, herramientas en Moodle, comunicación, control de aprendizaje, gestión de ePortfolio y redes sociales, entre otros); con la finalidad de administrar y gestionar entornos personales de aprendizaje acordes a los desafíos de la enseñanza actual. Esto coincide con Garduño (2005) quien afirma que la actual educación a distancia (virtual, remota) se apoya en medios generados a través de TIC y los datos hallados en los ítems 14, 15, 16, 17 y 18 de la (tabla 3), evidencian que las TIC son un medio para fortalecer el quehacer pedagógico, al adoptar el acceso a repositorios digitales y bases de datos, así como atender el uso de herramientas de comunicación asíncronas y síncronas (Prada, Hernández y Gamboa, 2019).

Esta adaptación al entorno digital puede implicar ajustes en las lecciones y promover estrategias de aprendizaje a través de comunicaciones móviles (aprendizaje-móvil), aprendizaje basados en la nube (que puede cambiar los modos de aprendizaje, o surgen oportunidades, mientras se aprende de la inteligencia colectiva en red a través de los nodos y conexiones de una red de aprendizaje que aumenta a medida que se interactúa en dicho entorno) mientras que el estudiante crea un sistema de aprendizaje cibernético en el hogar, adecuado a sus propias necesidades. Todo ello por una disposición a la plasticidad cerebral en la organización del trabajo y adaptación a las TIC fundado en una sociedad de la información y del conocimiento. 
Flexibilidad a las TIC en la enseñanza. La flexibilidad en la enseñanza no está establecida por la tecnología sino por motivaciones a las respuestas a estímulos externos bien conocidos en la educación, como las competencias TIC, aprendizaje en red, transformación a las demandas de la enseñanza en la era digital, cambios en la estructura de los procesos de enseñanza-aprendizaje, etc. Esto concuerda con el planteamiento de Arancibia (2011) quien sostiene que la flexibilidad se sustenta en la búsqueda de la adaptabilidad, que podría responder a una constante de modelos y tendencias educativas. Sin embargo, se deben mencionar dos características importantes de la flexibilidad a las TIC en la enseñanza: La primera, que concurre una interacción entre la innovación tecnológica y los cambios metodológicos de enseñanza. Las TIC arman y promueven la implementación de formas de enseñanza flexibles y desarrolla su eficiencia. La segunda, las TIC son una tecnología de gestión del tiempo y el espacio en la enseñanza, y otros tiempos sociales del aprendizaje, entre las esferas educador y estudiante.

Lo anterior coincide con lo propuesto por Piaget (1969) quien explica que la inteligencia ayuda a un individuo a adaptarse al entorno porque se halla una derivación del medio ambiente que lleva a un cambio flexible de adaptación que depende de la retroalimentación del entorno estimulante. Por ello, la flexibilidad a las TIC en la enseñanza, refiere a la capacidad de cambiar fácilmente a las nuevas formas de organizar, gestionar, orquestar y asegurar la enseñanza-aprendizaje mediadas por las tecnologías, la interacción entre diferentes actores y asignación de actividades, lo cual permite la capacidad para adaptarse, para adaptar, incluso para adoptar otro medio cambiando a él (Morín, 1980). Desde esta perspectiva, la flexibilidad respaldada por las TIC concuerda con los datos encontrados en los ítems 19, 20, 21, y 22 de la (tabla 3).

Además, ofrece oportunidades para el fortalecimiento de los procesos de enseñanza y de aprendizaje, sin importar el cuándo y dónde. Aunque la flexibilidad a las TIC en la enseñanza, obedece a una combinación de factores, como la afinidad del proceso de enseñanza-aprendizaje mediada por la tecnología, así como, la disponibilidad y calidad de su infraestructura tecnológica, la cultura de gestión y el impulso de una mayor productividad dentro de las organizaciones educativas, y las necesidades de autonomía de los docentes de adoptar las TIC por la flexibilidad espacial y temporal para la organización de actividades que susciten la praxis pedagógico como lo indica Lassos, Munévar, Rivera y Sabogal (2017) sobre realizar una estructuración de estrategias metodológicas que permitan el proceso formativo.

Adaptación a la enseñanza con TIC. Se refiere a la enseñanza virtual o remota con apoyo en las TIC, es decir, las metodologías, perspectivas, concepciones e intencionalidades que giran en torno a la docencia, y que, como resultado de la presión de las realidades configuradas por la pandemia, conllevan a cambios y ajustes variados para seguir llevando a cabo el acto de enseñanza. Es una experiencia con el medio circundante y de las interacciones con otros que llegan a incidir directamente en los esquemas dialógicos y la 
alteridad siempre presente en la pedagogía. Su sustento se encuentra en los hallazgos de los ítems 23 y 24 de la tabla 3, los cuales demuestran que los docentes estiman importante adaptarse a las TIC como apoyo didáctico en los procesos de enseñanza-. De esta manera, señalaron que piensan organizar cursos en línea, utilizar medios multimedios y hacer crear contenidos y materiales en línea.

En esta dirección, en la enseñanza se pueden distinguir dos dimensiones temporales en la adaptación ¿Cuáles? Cuando una persona aprende mediante las TIC, el entorno personal de aprendizaje comienza a crecer más rápido, se crean conexiones, los vínculos sociorelacionales se aceleran y, a medida que los vínculos se acumulan, se van explorando herramientas digitales y sitios web, las habilidades digitales e informacionales se vuelve importante para aprender. Este proceso es de adaptación como lo plantea Guzmán (2018), en el cual el ser humano modifica el comportamiento para acoplarse a nuevos procesos, para desarrollar la capacidad para acomodarse de forma eficiente y realizar las funciones sin que afecte las actividades. Por consiguiente, podría conseguir estabilidad (me ayuda tener ideas establecidas) y en otras situaciones es adaptativo el cambio (me ayuda cambiar mis ideas e intentar algo nuevo). Esto, podría llevar a una adaptación de los docentes a la enseñanza con TIC ante la emergencia educativa y está en concordancia con el planteamiento de Morín (1980) que permite aptitud de adaptación para adoptar y acoger otro medio cambiando a él.

Para concluir, es importante destacar que es indudable que el docente debe contar con las competencias digitales para aprovechar las herramientas y aplicarlas de forma tal que pueda obtener de ellas el mayor provecho posible del uso de las TIC en el proceso educativo. Sin embargo, la oportunidad que se le presenta al docente al utilizarlas para enseñar regularmente en tiempo de coronavirus podría generar que las habilidades para enseñar con TIC se vuelven más diestra y que toda la enseñanza virtual o mediada por TIC pareciera funcionar de manera más eficiente. En consecuencia, las competencias TIC podrían o deberían aumentar.

En este caso, el individuo (docente) hace una asimilación dentro de este proceso, mediante el cual interpreta sus nuevas experiencias, al tiempo que las adapta de manera adecuada a los patrones de pensamiento existentes. Por ello, hace una acomodación por la necesidad de cambiar los patrones de pensamiento existentes para ajustar la nueva información que simplemente no encajaría en el esquema anterior. Por consiguiente, el docente (individuo) se ha adaptado a la tarea descrita para cumplir mejor con los requisitos del hecho educativo mediado por las tecnologías. Todo para que la educación mediada por las TIC pueda garantizar el proceso necesario para la enseñanza-aprendizaje. Como se observa, este es un ajuste a corto plazo para el docente.

Pero estos ajustes a corto plazo develados entre los docentes como manifestación de su adaptación a una enseñanza apoyada o mediada por TIC, pueden configurarse como condiciones permanentes que no desaparecerían en un hipotético escenario de normalidad 
académica. En ese sentido, el docente desde las TIC, aprende, desaprende y reaprende en función de mejorar sus prácticas de enseñanza. Por tanto, se puede hablar de cambios estructurales en las formas de enseñar con apoyo de las TIC.

Entonces, en el primer caso, el uso de las TIC cambió dentro de los límites de sus capacidades tradicionales de la enseñanza, que terminaron tan pronto como desapareció el estímulo externo. En el segundo caso, las habilidades TIC del docente se podrían desarrollar a un nuevo nivel debido a cambios estructurales en las competencias del propio docente. De este modo, las actuales circunstancias en el marco de la pandemia pueden llevar a oportunidades que impliquen beneficios, por ejemplo, una mayor flexibilidad de los docentes respecto de la enseñanza que adelantan y todo lo que ello implica, además de habilidades potenciadas para el uso e incorporación de las TIC en lo educativo.

Por consiguiente, la autorregulación, los intereses y motivaciones de cada educador afectan o modifican sus procesos e intencionalidades de enseñanza, lo que lleva a una organización particular de los objetivos, saberes y metodologías. Se espera una mayor flexibilidad en estos esquemas para que con la articulación de enfoques, puedan dar respuesta a las situaciones y circunstancias cambiantes del entorno. Lo fundamental es estar abierto a diferentes ideas y perspectivas que haya en el futuro, oportunidades en la creación de ambientes más flexibles para el aprendizaje, aumento de los medios comunicativos, fortaleciendo los escenarios y entornos interactivos, así como mediar y guiar tanto en el aprendizaje activo, colaborativo como autodeterminado y rompiendo los tradicionales espacios docentes circunscritos a las instituciones educativas.

\section{Referencias}

Álvarez, V., Romero, S., Gil, J., Rodríguez, J., Clares, J., Asensio, I., del-Frago, R., García, B., García, M., González, D., Guardia, S., Ibarra, M., López, R., Rodríguez, G., Salmeron, P. (2011). Necesidades de formación del profesorado universitario para la adaptación de su docencia al Espacio Europeo de Educación Superior (EEES). RELIEVE, 17(1). https://doi.org/10.7203/relieve.17.1.4122

Arévalo, M., García, M. y Hernández, C. (2019). Competencias TIC de los docentes de matemáticas en el marco del modelo TPACK. Civilizar: Ciencias Sociales y Humanas, 19(36), 115-132. https://doi.org/10.22518/usergioa/jour/ccsh/2019.1/a07

Arancibia, F. (2011). Flexibilidad laboral: elementos teóricos-conceptuales para su análisis. Revista Ciencias Sociales (CI), (26), 39-55. http://www.revistacienciasociales.cl/archivos/revista26/pdf/rcs-art3.pdf

Barberousse, P. (2008). Fundamentos teóricos del pensamiento complejo de Edgar Morin. Revista Educare, $12(2)$,

95-113. http://www.redalyc.org/pdf/1941/194114586009.pdf

Bernal, C. (2010) Metodología de la Investigación. Para administración, economía, humanidades y ciencias sociales. Pearson Educación. 
Cabero, J. (2014). Reflexiones sobre la brecha digital y la educación: siguiendo el debate. Inmanencia, $4(2)$, http://ppct.caicyt.gov.ar/index.php/inmanencia/article/view/6242

14-26.

Cabero, J., López, E. y Martínez, A. (2013). Los portafolios educativos virtuales en las aulas universitarias. instrumentos didácticos para la innovación docente y la calidad de los procesos de enseñanza y aprendizaje. Enseñanza \& Teaching: Revista $\begin{array}{llll}\text { Interuniversitaria De Didáctica, 31(1), 43-70. } & \text {. }\end{array}$ https://revistas.usal.es/index.php/0212-5374/article/view/11604

Del Moral, M. y Villalustre, L. (2005). Adaptación de los entornos virtuales a los estilos cognitivos de los estudiantes: un factor de calidad en la docencia virtual. Píxel-Bit. Revista de Medios y Educación, (26), 16-25. https://recyt.fecyt.es/index.php/pixel/article/view/61257

Díaz, A, Pérez, M, Valenzuela, M., Muñoz, P., Rivas, S. y Salas, C. (2010). Procesos de autorregulación del aprendizaje en estudiantes universitarios de primer año. International Journal of Developmental and Educational Psychology, 4(1), 789-800. https://www.redalyc.org/articulo.oa?id=3498/349832327082

Gamboa, A., Hernández, C. y Prada, R. (2018). Práctica pedagógica y competencias TIC. Saber, Ciencia y Libertad, 13(1), 258-274. https://doi.org/10.18041/23823240/saber.2018v13n1.2090

García, L. (2002). La educación a distancia. De la teoría a la práctica. Ariel.

Garduño, V. (2005). Enseñanza virtual sobre la organización de recursos informativos digitales. UNAM, Centro Universitario de Investigaciones Bibliotecológicas.

Goh, P. \& Sandars, J. (2020). A vision of the use of technology in medical education after the COVID-19 pandemic. MedEdPublish, 9(1), 49, https://doi.org/10.15694/mep.2020.000049.1

Gómez, C., Hernández, C. y Prada, R. (2020). La zona de posibilidades en el proceso de aprendiencia del residente digital: un análisis cualitativo en la red de experiencias matemáticas de Norte de Santander. Educación y Humanismo, 22(38), 1-19. https://doi.org/10.17081/eduhum.22.38.3688

Guzmán, A. (2017). Brecha digital de las herramientas web 2.0, entre los docentes de la Institución Educativa Agropecuaria de Fonseca. Telematique, 16(1), 43-59. http://ojs.urbe.edu/index.php/telematique/article/view/2610

Guzmán, A. (2018). Adaptabilidad y proceso de cambio organizacional. [Tesis de grado, Universidad Rafael Landívar, Quetzaltenango, México]. http://recursosbiblio.url.edu.gt/tesiseortiz/2018/05/43/Guzman-Andrea.pdf

Guzmán, T., García, M., Espuny, C. y Chaparro, R. (2011). Formación docente para la integración de las TIC en la práctica educativa. Apertura, 3(1), 6-13. http://www.udgvirtual.udg.mx/apertura/index.php/apertura/article/view/181/196

Hernández, C., Arévalo, M. y Gamboa, A. (2016). Competencias TIC para el desarrollo profesional docente en educación básica. Praxis \& Saber, 7(14), 41 - 69. https://doi.org/10.19053/22160159.5217

Jaume, A. (2011). Adaptabilidad y funcionalidad: una teoría acerca de las funciones biológicas. Ludusvitalis: Revista de filosofía de las ciencias de la vida. 19(35), 97- 


\section{2. http://ludus-vitalis.org/html/textos/35/35_05_jaume.pdf}

Jojoa, H. (2014). Adaptabilidad de la estrategia de indagación a través de plataforma virtual UDENAR para mejorar la competencia crítica y autocrítica, aplicada a estudiantes de pregrado de la Universidad de Nariño [tesis de Maestría, Universidad de Nariño, Pasto Colombia]. http://sired.udenar.edu.co/1839/

Lassos, E., Munévar, P., Rivera, J. y Sabogal, A. (2017). Estado del arte sobre la articulación de modelos enfoques y sistemas en educación virtual. Sello Editorial UNAD. https://hemeroteca.unad.edu.co/index.php/book/issue/view/196

Loredo, J. (2004). La teoría de la selección orgánica de Baldwin y la escisión entre naturaleza y cultura. Acción psicológica, 3(3), 187-198. http://revistas. uned.es/index.php/accionpsicologica/article/download/512/451

Martín, M., Hernández, C. y Mendoza, S. (2017). Ambientes de aprendizaje basados en herramientas web para el desarrollo de competencias TIC en la docencia. Revista Perspectivas, 2(1), 97-104. https://doi.org/10.22463/25909215.1282

Morin, E. (1980). El Método II. La vida de la vida. Seuil.

Piaget, J. (1969). Psicología y Pedagogía. Ariel.

Prada, R., Hernández, C. y Gamboa, A. (2019). Usos y efectos de la implementación de una plataforma digital en el proceso de enseñanza de futuros docentes en matemáticas. Revista Virtual Universidad Católica del Norte, (57), 137-156. https://doi.org/10.35575/rvucn.n57a10

Prensky, M. (2001). Nativos digitales, inmigrantes digitales. Onthehorizon, 9(6), 1-7. http://files.educunab.webnode.cl/200000062-5aba35bb22/Nativos-digitalesparte1.pdf

Sánchez, R., Costa, O., Mañoso, L., Novillo, M. y Pericacho, F. (2019). Orígenes del conectivismo como nuevo paradigma del aprendizaje en la era digital. Educación y Humanismo, 21(36), 113-136. http://dx10.17081/eduhum.21.36.3265

Siemens, G. (2004). Connectivism: A learning theory for the digital age. International Journal of Instructional Technology and Distance Learning, (5), 1-6. http://citeseerx.ist.psu.edu/viewdoc/download?doi=10.1.1.87.3793\&rep=rep1\&typ $\mathrm{e}=\mathrm{pdf}$

UNESCO-IESALC (2020). COVID-19 y educación superior: Análisis de impactos, respuestas politicas y recomendaciones. http://www.iesalc.unesco.org/wpcontent/uploads/2020/04/COVID-19-060420-ES-2.pdf

White, D. \& Le Cornu, A. (2011). Visitors and Residents: A new typology for online engagement. First Monday,

http://firstmonday.org/article/view/3171/3049 